\title{
Characterization, slab-pair modeling and phase analysis of circular fishnet metamaterials
}

\author{
Yusuf Öztürk $^{\mathrm{a}, \mathrm{b}, *}$, Asım Egemen Yılmaz ${ }^{\mathrm{a}}$, Evrim Çolak ${ }^{\mathrm{c}}$, Ekmel Özbay ${ }^{\mathrm{c}}$ \\ ${ }^{a}$ Ankara University, Department of Electrical and Electronics Engineering, Tandoğan, 06100 Ankara, Turkey \\ ${ }^{\mathrm{b}}$ TÜBİTAK-ULAKBIM, Bilkent, 06539 Ankara, Turkey \\ ${ }^{\mathrm{c}}$ Nanotechnology Research Center, Bilkent University, Bilkent, 06800 Ankara, Turkey
}

Received 9 March 2012; received in revised form 29 May 2012; accepted 29 May 2012

Available online 9 June 2012

\begin{abstract}
Planar metamaterials, which have incident to normal plane excitation unlike SRR-type structures and that are easily fabricated in multilayer form, have received great interest in recent years. In this paper, one-dimensional and polarization independent circular fishnet metamaterials and their equivalent discontinuous slab-pair modeling for tuning resonance frequencies are introduced. After the numerical and experimental demonstration of the inclusions, the standard retrieval characterization methods and the correspondent/related backward-wave propagation observation are realized in order to check the physical explanation mentioned in the paper. In addition, a detailed phase analysis is performed in order to demonstrate the application of the suggested structure as a phase compensator.
\end{abstract}

(C) 2012 Elsevier B.V. All rights reserved.

Keywords: Circular fishnet metamaterials; Double negative (DNG) metamaterials; Slab-pair modeling; Phase compensation

\section{Introduction}

The phenomenon of artificial engineered metamaterials that cannot be obtained with naturally occurring materials was initiated with the theoretical consideration of the negative refraction concept by Veselago [1]. An experimental demonstration of the medium, which was called as electromagnetic metamaterial, was realized by using split ring resonators (SRRs) and continuous wires [2] following the ideas described by Pendry et al. In these studies, the periodical arrangement of thin wires provides a negative effective

\footnotetext{
* Corresponding author at: TÜBITAK-ULAKBİM, Bilkent, 06539 Ankara, Turkey. Tel.: +90 5324782678.

E-mail address: yusuf.ozturk@tubitak.gov.tr (Y. Öztürk).
}

permittivity at frequencies lower than the plasma frequency of the system [3]. The SRRs, as microstructures built from nonmagnetic conducting sheets, provide a strong magnetic resonance, and exhibit an effective magnetic permeability [4]. Due to difficulties with fabrication of SRRs with the resonance frequency at the visible range and the alignment of the produced layers for multilayer applications, the introduction of planar metamaterials were suggested to overcome the restrictions involving conventional metamaterials [59]. Moreover, split ring resonators are not suitable for the planar utilization of metamaterials, since the incident wave has to be parallel to the SRR, which means that the magnetic field is perpendicular to the SRR. To satisfy optical magnetism, those alternative structures of metal-dielectric composites are made up with a dielectric substrate between parallel metal slabs 
providing the inductance and capacitance, simultaneously. On the other hand, fishnet-type metamaterials have been gaining wide popularity due to their polarization-independent characteristics for the nearinfrared [10] and microwave regime [11-15] of the electromagnetic spectrum. One of the simplified structures consists of periodical arrays of pairs of short metal wires and continuous wires offering a potentially simpler approach for achieving negative index materials [16]. During the characterization of metamaterials, retrieval methods have a wide usage in presenting the effective permittivity and permeability $[17,18]$. On the other hand, broadband phase shifters/compensators $[19,20]$ and left-handed medium (LHM)-right-handed medium (RHM) resonators [21] are some examples of potential applications of the metamaterials.

\section{Structure definition}

In this study, circular fishnet metamaterial (CF-MM), which is a structure having incident polarization independence, is investigated. For this purpose, after its design and fabrication, the structure is measured for complete behavioral characterization. Polarization independence of the structure is due to its symmetric configuration. A schematic view of the unit cell, multilayer, and equivalent slab pair forms of CF-MMs are depicted in Fig. 1. The structure consists of the lowloss Teflon substrate (with $\varepsilon_{r}=2.16$ and loss tangent of $\delta=0.0004$ ) with a transparent view, and the highlighted metal parts (copper) as a PEC layer. Low loss tangent value is vital for achieving left-handed resonance behavior, and therefore, FR-4 like substrates are not suitable for creating CF-MM with the same or comparable dimensions. The Teflon layer as a substrate and the copper layer have thicknesses of $t=1 \mathrm{~mm}$ and $20 \mu \mathrm{m}$, respectively. The unit cell shown in Fig. 1(a) has complementary parameters wherein choosing the dimensions as $a_{x}=a_{y}=a=14 \mathrm{~mm}$ and the radius $r=0.25 \times a$ in turn would result in fully circular and polarization-independent inclusions. The unit cell is replicated nine times in both $x$ and $y$ directions in order to obtain the whole singlelayer CF-MM structure with $10 \times 10$ cells. For the multilayer deployment of CF-MMs as shown in Fig. 1(b), the distance between the stacked layers equals $a_{z}=2 \mathrm{~mm}$. The incident EM wave propagates along the $z$-direction perpendicular to the $E$-field (which is parallel to the $y$ axis) and the $H$-field (which is parallel to the $x$-axis).

First, we investigate the resonance behavior of the structure in order to develop a simple method satisfying the desired operation conditions. Taking Fig. 1(c) into account, the equivalent model of CF-MMs could be handled as a discontinuous slab-pair or a cut-wire pair form. Negative dielectric permittivity is easily obtained with a system of such parallel wires and exhibits Drudelike plasmonic behavior for the frequencies below plasma-frequency $\left(\omega_{p}\right)$, while negative permeability in all these structures creates a strong magnetic resonance at a frequency $\omega=\omega_{m}$ by exciting resonant circular currents; this basic process can be simulated by an effective $L C$-circuit exhibiting a resonance at $\omega_{m}=\sqrt{L C}$. Similar to what is identified in [16], the inductance value $L$ and the capacitance value $C$ can be computed by using $L=\mu_{0}\left(l_{\text {eff }} \cdot t\right) / w_{\text {eff }}$ and $C=\varepsilon_{r} \varepsilon_{0}\left(l_{\text {eff } f} \cdot w_{\text {eff }}\right) / 4 t$, where $l_{\text {eff }}$ and $w_{\text {eff }}$ are the effective length and width as depicted in Fig. 1(c), $t$ is the thickness of the substrate, $\mu_{0}$ is permeability in vacuum, $\varepsilon_{0}$ is permittivity in vacuum and $\varepsilon_{r}$ is the relative dielectric constant of the Teflon substrate. These equations result in the DNG or LHM magnetic resonance frequency $\left(f_{m}\right)$ as:

$$
f_{m}=\frac{1}{2 \pi \sqrt{L C}}=\frac{c_{0}}{\pi l_{e f f \sqrt{\varepsilon_{r}}}}
$$

where $c_{0}$ is the speed of light in vacuum. According to Eq. (1), the magnetic-resonance frequency does not
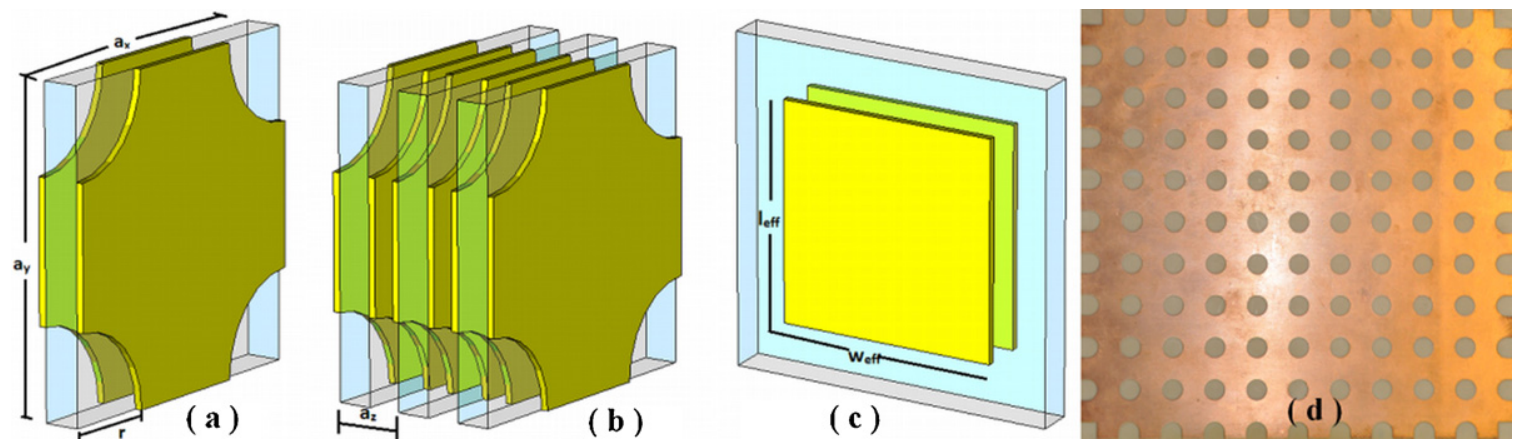

Fig. 1. Schematic representation of CF-MMs as (a) unit-cell, (b) multi-layer form of the unit-cells, (c) equivalent slab-pair form, and (d) the photography of the fabricated $10 \times 10$ cell CF-MM structure. 

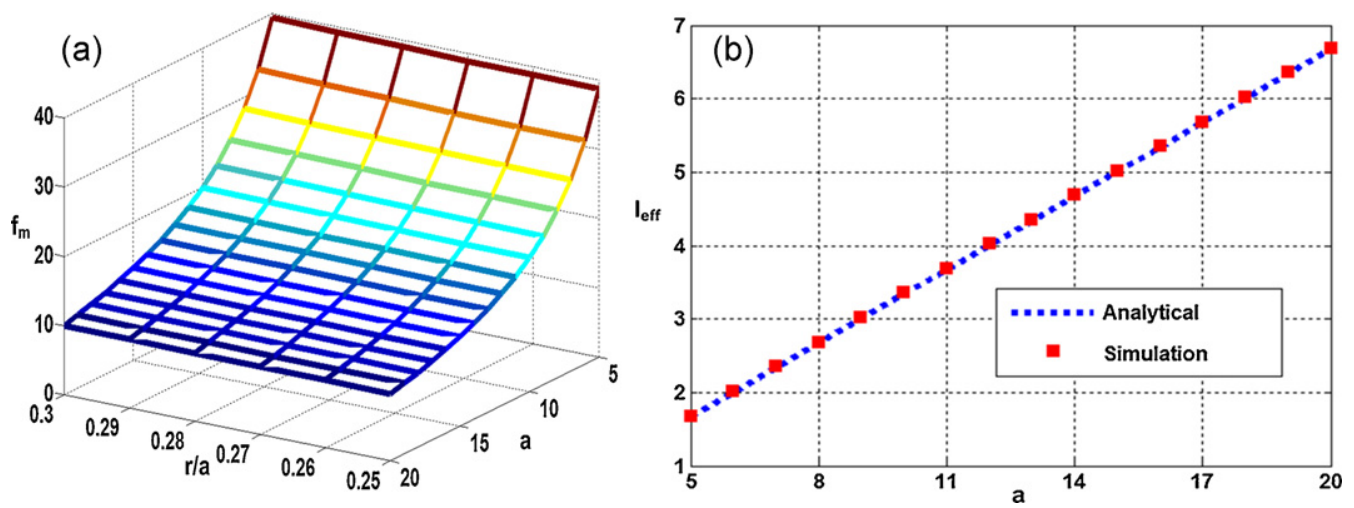

Fig. 2. Mesh view of the LHM resonance frequencies with respect to $r / a$ and $a$ (a). The results belonging to the discontinuous slab-pair modeling of CF-MM structure for $r=0.25 \times a$ (b).

depend on the parameters $t$ and $w_{\text {eff }}$. In fact, due to symmetry, it could be easily stated that the effective length of the unit-cell must be equal to the effective width of the unit-cell, namely $l_{\text {eff }}=w_{\text {eff }}$. In this situation, the thickness $(t)$ is the unique parameter independent of $f_{m}$ and only contributes the RHM resonance frequency. Additionally, the thickness affects the LHM transmission peak level based on the loss tangent value.

In fact, the resonance values could be calculated by using a full-wave simulator tool, alternatively. Fig. 2(a) draws a wireframe mesh using the unit cell length $r / a$ and the ratio of the radius to the unit cell length $a$ and the height corresponds to the LHM resonance frequencies manually selected during simulations. In this study, the scalability of CF-MMs is verified for wide-range unitcell dimensions from $5 \mathrm{~mm}$ up to $20 \mathrm{~mm}$ and the ratio from 0.25 to 0.30 . On the other hand, the substrate thickness is kept constant at $1 \mathrm{~mm}$ during the scale-up/ down process. As an example, the correspondent LHM resonance frequencies are between $9.7 \mathrm{GHz}$ and $38.74 \mathrm{GHz}$ for $r=0.25 \times a$. After the simulation process, the effective length values are calculated by using the selected frequencies based on Eq. (1). In this study, we introduce a relationship between the effective length and the unit cell length with linear approximation. As shown in Fig. 2(b), we analytically identified the effective length of the unit cell as $l_{\text {eff }} \approx 0.334 a$ with a very-good agreement with the simulations for $r=0.25 \times a$ and realized the discontinuous slab-pair modeling of CF-MMs. The effective lengths of other unit cells (which are designed for different $r / a$ values) can be computed via Eq. (1) inserting new resonance frequencies $f_{m}$. By means of such a model as a design guideline, for a fixed $r / a$ ratio, it is now possible to tune the resonance frequency to the desired region in the electromagnetic spectrum analytically. Additionally, availability of this technique is tested and verified for the squared-type fishnet metamaterials introduced in [14].

The metamaterial is designed and characterized by using the commercial software package, CST Microwave Studio. Fig. 3 shows the qualitative analysis of CF-MM structures in the backward and forward propagation cases. In Fig. 3(a), from the top view of the unit cell ( $y z$-plane), it is clear to observe the omitted fringing electric field intensities; whereas Fig. 3(b) holds those values along the circular sides of the unit cell. As a physical explanation, we focused on the relationship between the electrical permittivity $\varepsilon_{r}$ and the electric polarization $P$ with the basic formula of $D=\varepsilon_{0} E+P_{e}=\left(1+\chi_{e}\right) \varepsilon_{0} E$. For $\chi_{e}<-1$ case, $\varepsilon_{r}$ becomes negative, satisfying the homogenization subwavelength conditions. This causes the electric polarization $P$ to overcome the electric flux density term, which is $\varepsilon_{0} E$. The same approach can be used in order to analyze the relationship between the effective magnetic susceptibility $\chi_{m}$ and the magnetic flux density $B$ with the second complementary formula $B=\mu_{0}\left(H+P_{m}\right)=\left(1+\chi_{m}\right) \mu_{0} H$. The cancellation of the horizontal electric field components produced by circular slots and the corresponding anti-parallelism in the surface currents are shown in Fig. 3(a) and (c), respectively. On the other hand, the fringing electric fields and the relevant parallel surface currents are depicted in Fig. 3(b) and (d). In this study, the longitudinal ( $z$-directed) TE or TM electromagnetic field is excited such that $a_{z} \ll \lambda$ (the operating wavelength) takes place during the whole characterization process. The CF-MM type structure has uniaxial properties (one-dimensional) and this limitation is satisfied with $a_{z}=\lambda / 11.5 \sim \lambda / 7.5$. To sum up, the magnetic field perpendicular to the electric field at every 


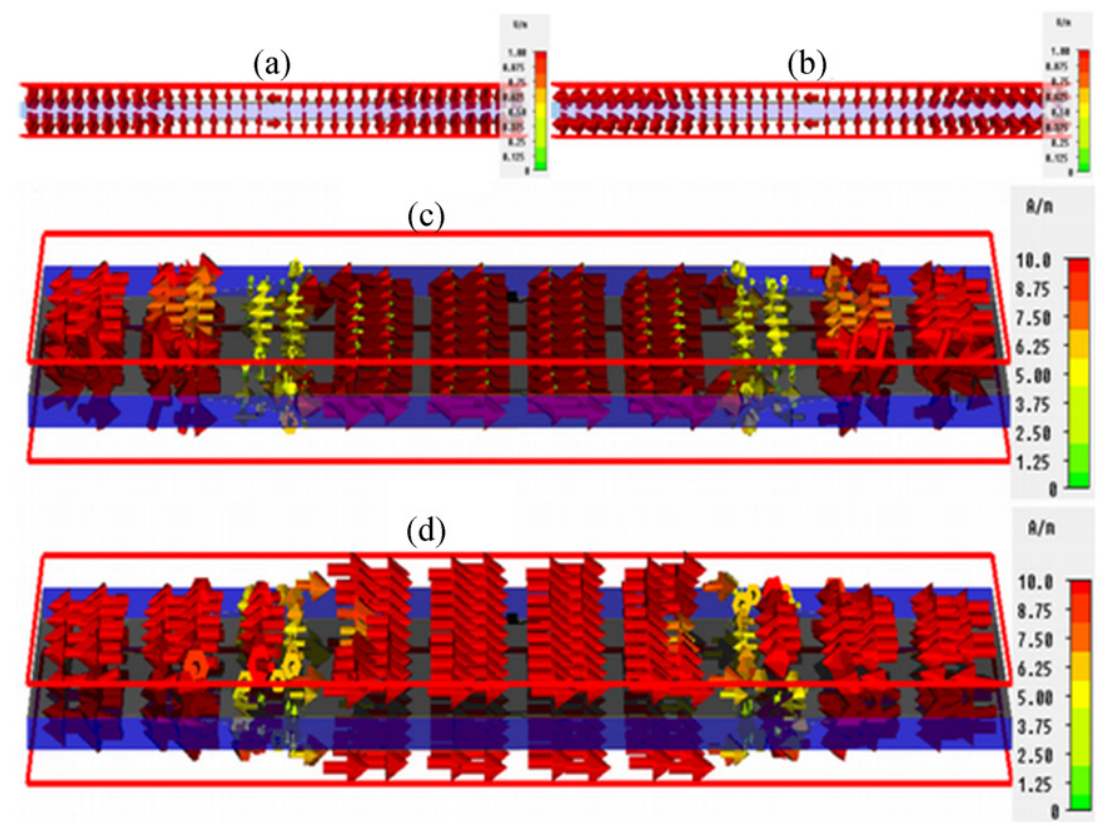

Fig. 3. Top view of the calculated electric field intensity values (E) in V/m for LHM (in (a)) and RHM (in (b)). At the bottom (in (c) and (d)), the corresponding surface currents in $\mathrm{A} / \mathrm{m}$ are presented. The PEC parts of the structures are presented with full transparency for better visualization.

point in space has the impact of creation of a circular field surrounding the necks of the structure for RHM investigation. In this situation, the magnetic field in the apertures of the structure consisting of only dielectric layer has negligible importance. On the other hand, cancellation of the fringing electric field yields in comparable magnetic field around the apertures. This vanishes the circularity of the magnetic field surrounding the necks of the structure. Under these homogenized medium conditions, surface currents show a continuous movement along the unit cell sheets for the LHM region, and a reversal discrete behavior in the RHM region.

The standard retrieval procedure [17] was implemented before the CF-MM structures were manufactured and measured. For this purpose, a unit-cell is employed along the $z$-direction; the effective permittivity and permeability values were then derived from the magnitudes and phases of the transmission/ reflection coefficients $\left(S_{21}\right.$ and $\left.S_{11}\right)$ of a single-layer CF-MM seen in Fig. 4(a). As shown in Fig. 4(b), the double negative (DNG) region is created by the negative permeability boundaries of $13.75-14.1 \mathrm{GHz}$, which is due to the availability of the negative permeability for the entire frequency range below the plasma frequency $\left(\omega_{p}\right)$ originating from Drude like plasmonic behavior $\varepsilon(\omega)=1-\omega_{p}^{2} / \omega^{2}$. The double positive (DPS) medium is located in a relatively wider bandwidth according to the DNG medium above $19 \mathrm{GHz}$. Under the conditions $\operatorname{Re}(\varepsilon)<0$ and $\operatorname{Re}(\mu)<0$, which hold for the frequency interval of $13.75-14.1 \mathrm{GHz}$, LHM behavior is expected. However, there is another factor affecting electromagnetic wave propagation in the $+z$ or $-z$ direction; the imaginary parts of the refractive index. It is clear to identify that the imaginary parts attain zero-value for the resonance frequencies of the LHM and RHM regions, centered at 13.8 and $19.5 \mathrm{GHz}$. By combining all the information, we expect a band spectrum narrower than a $13.5-14.1 \mathrm{GHz}$ interval. For different designs needing a wider DPS interval, one should either increase the unit cell radius, or use the multilayer form of the CF-MM inclusions.

\section{Experimental results}

The experiments are performed in free space and at room temperature by using two standard-gain horn antennas. The distance between the antennas was kept fixed at $30 \mathrm{~cm}$, and the specimen (unit cell) was located at the center. First, a TRL calibration procedure was implemented on the network analyzer in order to eliminate the environmental noises. After the free-space calibration, the transmission spectra and phase spectra have been measured at the same position.

The measured and calculated transmission and reflection characteristics of the CF-MM medium are 

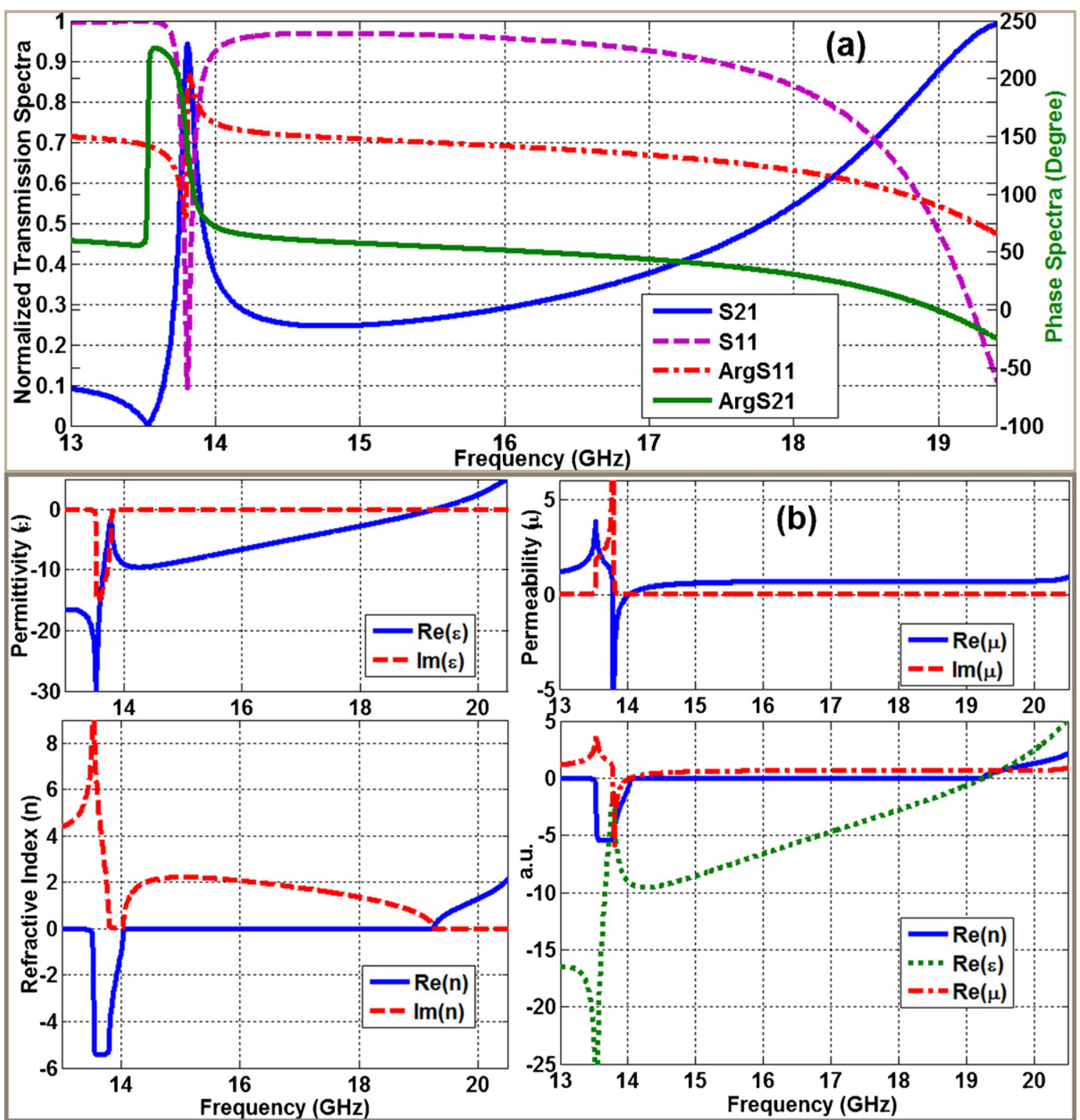

Fig. 4. (a) The computed magnitudes and phases of the scattering parameters $\left(\left|\mathrm{S}_{11}\right|,\left|\mathrm{S}_{21}\right|, \operatorname{Arg} \mathrm{S}_{11}, \operatorname{Arg} \mathrm{S}_{21}\right)$ used for the retrieval analysis. (b) The retrieved effective parameters for the CF-MMs. The solid blue and dashed red lines show the real parts of the refractive index $(n)$, the relative permittivity $\left(\varepsilon_{r}\right)$, and the permeability $\left(\mu_{r}\right)$. (For interpretation of the references to color in this figure legend, the reader is referred to the web version of the article.)

displayed in Fig. 5. The data show compatibility with the outputs of the standard retrieval procedure as depicted in Fig. 4; where the first resonance frequency for the LHM is observed at $13.8 \mathrm{GHz}$, and the second resonance frequency for the RHM takes place at 19.2 GHz. For this type of metamaterial, there are basically three mechanisms resulting in undesired losses. The loss-tangent value of the dielectric substrate has a strong effect on the peak value of LHM resonance frequency. A small amount of degradation from the ideal value causes attenuation for the peak values of the very narrow LHM band. The best measurement for the LHM resonance in our experiment equals to $-4.8 \mathrm{~dB}$. The second loss mechanism related to the RHM resonance frequency is the thickness of imperfect conductors, which are modeled as PEC during the simulations. Increased metal thickness results in a loss in the RHM resonance peak value; and a metal thickness 

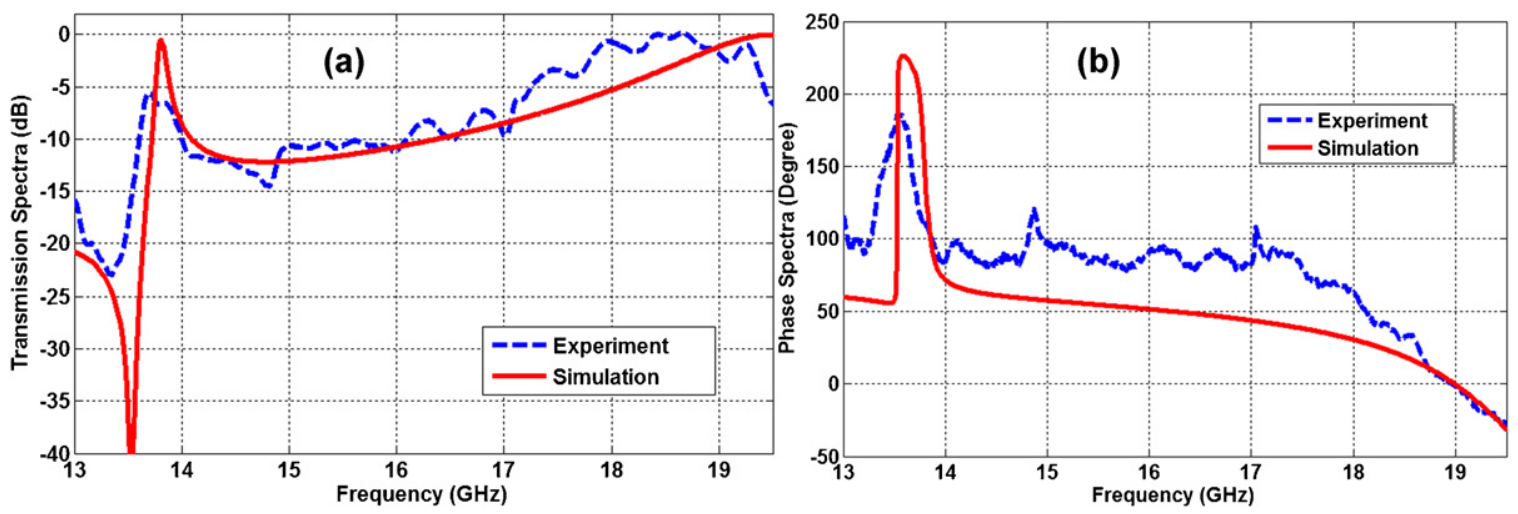

Fig. 5. Calculated (red solid curve) and measured (blue dashed curve) transmission and unwrapped phase spectra values for single-layer CF-MM in $\mathrm{a}$ and $\mathrm{b}$, respectively. (For interpretation of the references to color in this figure legend, the reader is referred to the web version of the article.)

less than $20 \mu \mathrm{m}$ shifts the RHM resonance location to lower frequency values. As a result, it is highly probable that the fabricated specimen has a loss tangent value greater than 0.0004 , and a metal thickness less than $20 \mu \mathrm{m}$ due to various uncontrollable effects such as corrosion. On the other hand, the measured phase spectra are compatible with the calculated phase spectra in the characteristic features except for an offset value. We observed phase advance behavior in the LHM region and linear phase lag in the RHM region. In comparison to the RHM phase lag, the phase advance occurs in a more steeper and narrow shape based on what is depicted in the retrieval values of $n$.

\section{Theoretical multilayer phase analysis of CF-MMs}

We finally analyzed the transmitted phase of CFMMs in order to investigate the change in the phase velocities for the multi-layer form of the structure. As expected, the medium with $\varepsilon<0$ and $\mu<0$ shows a phase advance rather than a phase lag for the ordinary materials. Thus, the more layers of the negative index material, the more phase advance exists in the LHM region as well as the more phase lag in the RHM region. The index of refraction (in terms of wavelength, phase shift, and the length-change of the material in the propagation direction) is given by [22]:

$n=\frac{\Delta \phi}{\Delta L} \frac{\lambda}{2 \pi}$

Before the implementation of Eq. (2), we performed a pre-processing study to obtain the absolute phase shift that is defined as the phase difference in the calculated phase value for port-to-port and the air-layer between the ports. This relationship is defined in Eqs. (3a) and (3b):

$\Delta \phi_{\text {total }}=\left|n_{1}\right| k_{0} d_{1}-n_{2} k_{0} d_{2}$

$\Delta \phi_{\text {total }}=\Delta \phi_{\mathrm{CF}-\mathrm{MM}}-\Delta \phi_{\text {air }}$

where $n_{1}$ and $d_{1}$ denote the refractive index and the material thickness of CF-MMs, respectively. On the other hand, $n_{2}$ and $d_{2}$ represent the refractive index and the thickness of the air between the ports used for transmitter and receiver devices, where $n_{2}=1 \mathrm{~mm}$ and $d_{2}=30 \mathrm{~mm}$. It should be noted that, for specific applications, in order to decrease the thickness (namely the $d_{2}$ value), another material having refractive index value more than unity could be used.

At first glance, Fig. 6(a) seems to be a little bit confusing if one makes a connection between these phase spectra and the results of the standard retrieval procedure or the transmission spectra depicted in Fig. 4. First of all, the backward wave propagation starts from 13.5 GHz, a dip point in the transmission spectra, and continues up to the resonance frequency. Similar to the LHM region, the forward propagation mechanism is produced by passing the second dip value near to $14.2 \mathrm{GHz}$ with maximum attenuation behavior. As a result, the phase analysis studies give us an opportunity to identify the boundaries of the LHM and RHM regions clearly.

Phase compensators are designed to perform the partial or complete removal of phase shift of electromagnetic wave propagating through a structure containing both positive and negative refractive index material. One of the simplest structures to achieve this kind of a device is the combination of two-sequential slabs containing LHM and RHM media in each slab 

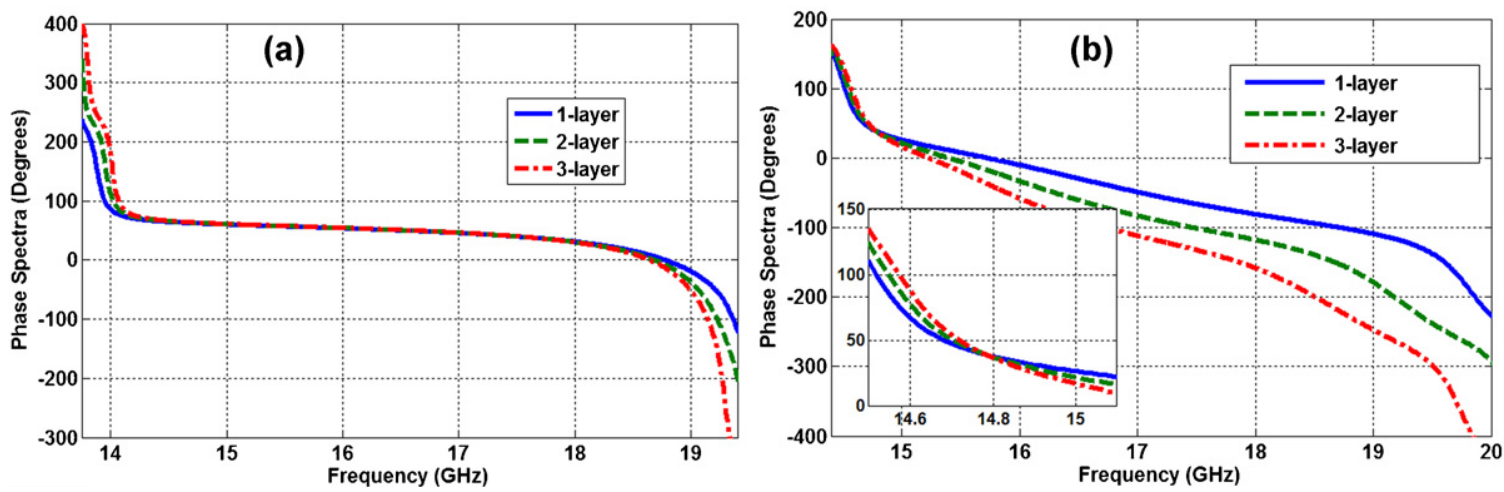

Fig. 6. Unwrapped transmitted phase spectra belonging to the multi-layer CF-MMs with $r / a=0.25$ (in a) and $r / a=0.33$ (in b). The inline picture represents a closer look at the transition between the LHM and RHM regions.

simultaneously. Focusing on Eq. (3a), the thicknesses of each slab depend on the refractive index value of the other slab expressed as $\left|n_{1}\right| / n_{2}=d_{2} / d_{1}$. However, by taking the previous works into account, we introduce some difficulties during the implementation of this relationship. In Fig. 6(a), a nearly constant phase shift/ difference could be handled for every additional layer at a fixed frequency above $19 \mathrm{GHz}$; whereas, it is not possible for the LHM slab in the $13.75-14.1 \mathrm{GHz}$ regime. The main reason for this problem is the decrease of the negative refractive index value obtained from the thicker LHM slab. In previous studies, this change of the negative refractive index was explained with a Figure of Merit (FOM) expressed as FOM= $|\operatorname{Re}(n)| / \operatorname{Im}(n)$. The equilibrium state between the slab thickness and the negative refractive index causes a relatively small phase difference in comparison with the RHM slab.

On the other hand, we deployed a different configuration of CF-MMs as depicted in Fig. 6(b). The change in the $r / a$ value not only shifts the resonance frequency $\left(f_{m}\right)$ up to $14.6 \mathrm{GHz}$, but also affects the dispersion characteristics of the structure. The configuration with $r / a=0.25$ (having a band-stop behavior between the LHM and RHM regions) results in an unmatched case for transmission line modeling, whereas the other configuration with $r / a=0.33$ shows a matched behavior or a direct transition between the LHM and RHM regions at $14.8 \mathrm{GHz}$. By taking this detail into account, the systematic characterization process outlined in this paper can be applied to different versions of CF-MMs.

\section{Conclusion}

In summary, we designed and fabricated a circular fishnet-type negative refractive material and performed experiments to observe the exact and real behavior of our structure. The simulation with full-wave analysis and experimental results for the scattering parameters $\left(S_{21}\right.$ and $\left.S_{11}\right)$ show good agreement with acceptable frequency shifts. In this paper, a direct relationship between anti-parallel surface currents and the cancellation of the fringing electric field strength is explained in order to clarify the physical mechanism behind the negative refraction phenomenon. In addition, an equivalent discontinuous slab-pair modeling for this type of metamaterial is proposed in order to describe a guideline for tuning the resonance frequency while the sub-wavelength criterion is being considered. Moreover, we realized a detailed phase analysis for the complete-regime of the implementation by making a cross-examination of our results and previous studies. The backward and forward propagation behavior has been observed as a successful implementation of the stacked multilayer form of CF-MMs.

\section{References}

[1] V.G. Veselago, Electrodynamics of substances with simultaneously negative electrical and magnetic permeabilities, Soviet Physics Uspekhi 10 (1968) 509.

[2] D.R. Smith, W.J. Padilla, D.C. Vier, S.C. Nemat-Nasser, S. Schultz, Composite medium with simultaneously negative permeability and permittivity, Physical Review Letters 84 (2000) 4184-4187.

[3] J.B. Pendry, A.J. Holden, W.J. Stewart, I. Youngs, Extremely low frequency plasmons in metallic mesostructures, Physical Review Letters 76 (1996) 4773-4776.

[4] J.B. Pendry, A.J. Holden, D.J. Robbins, W.J. Stewart, Magnetism from conductors and enhanced nonlinear phenomena, IEEE Transactions on Microwave Theory and Techniques 47 (1999) 2075-2084.

[5] C. Enkrich, M. Wegener, S. Linden, S. Burger, L. Zschiedrich, F. Schmidt, J.F. Zhou, T. Koschny, C.M. Soukoulis, Magnetic metamaterials at telecommunication and visible frequencies, Physical Review Letters 95 (2005) 203901. 
[6] M.W. Klein, C. Enkrich, M. Wegener, C.M. Soukoulis, S. Linden, Single-slit split-ring resonators at optical frequencies: limits of size scaling, Optics Letters 31 (2006) 1259-1261.

[7] S. Zhang, W. Fan, N.C. Panoiu, K.J. Malloy, R.M. Osgood, S.R.J. Brueck, Experimental demonstration of near-infrared negative-index metamaterials, Physical Review Letters 95 (2005) 137404.

[8] V.M. Shalaev, W. Cai, U.K. Chettiar, H. Yuan, A.K. Sarychev, V.P. Drachev, A.V. Kildishev, Negative index of refraction in optical metamaterials, Optics Letters 30 (2005) 3356-3358.

[9] G. Dolling, G. Enkrich, M. Wegener, J.F. Zhou, C.M. Soukoulis, S. Linden, Cut-wire pairs and plate pairs as magnetic atoms for optical metamaterials, Optics Letters 30 (2005) 3198-3200.

[10] K.B. Alici, E. Ozbay, Characterization and tilted response of a fishnet metamaterial operating at $100 \mathrm{GHz}$, Journal of Physics D: Applied Physics 41 (2008) 135011.

[11] M. Kafesaki, I. Tsiapa, N. Katsarakis, T. Koschny, C.M. Soukoulis, E.N. Economou, Left-handed metamaterials: the fishnet structure and its variations, Physical Review B 75 (2007) 235114.

[12] V.D. Lam, J.B. Kim, S.J. Lee, Y.P. Lee, Left-handed behavior of combined and fishnet structures, Journal of Applied Physics 103 (2008) 033107.

[13] J. Zhou, T. Koschny, M. Kafesaki, C.M. Soukoulis, Size dependence and convergence of the retrieval parameters of metamaterials, Photonics and Nanostructures: Fundamentals and Applications 6 (2008) 96.
[14] K. Aydin, Z. Li, L. Sahin, E. Ozbay, Negative phase advance in polarization independent, multi-layer negative-index metamaterials, Optics Express 16 (2008) 8835-8844.

[15] K.B. Alici, E. Ozbay, A planar metamaterial: polarization independent fishnet structure, Photonics and Nanostructures: Fundamentals and Applications 6 (2008) 102-107.

[16] J. Zhou, L. Zhang, G. Tuttle, T. Koschny, C.M. Soukoulis, Negative index materials using simple short wire pairs, Physical Review B 73 (2006) 041101(R).

[17] X. Chen, T.M. Grzegorczyk, B.-I. Wu, J. Pacheco Jr., J.A. Kong, Robust method to retrieve the constitutive effective parameters of metamaterials, Physical Review E 70 (2004) 016608.

[18] D.R. Smith, S. Schultz, P. Markos, C.M. Soukoulis, Determination of effective permittivity and permeability of metamaterials from reflection and transmission coefficients, Physical Review B 65 (2002) 195104.

[19] Z. Jakšić, N. Dalarsson, M. Maksimović, Negative refractive index metamaterials: principles and applications, Microwave Review 12 (2006) 36.

[20] I.S. Nefedov, S.A. Tretyakov, On potential applications of metamaterials for the design of broadband phase shifters, Microwave and Optical Technology Letters 45 (2005) 98.

[21] Y. Li, L. Ran, H. Chen, J. Huangfu, X. Zhang, K. Chen, T.M. Grzegorczyk, Experimental realization of a one-dimensional LHM-RHM resonator, IEEE Transactions on Microwave Theory and Techniques 53 (2005) 1522-1526.

[22] K. Aydin, K. Guven, C.M. Soukoulis, E. Ozbay, Observation of negative refraction and negative phase velocity in left-handed metamaterials, Applied Physics Letters 86 (2005) 124102. 